\title{
Flavin-containing monooxygenases: mutations, disease and drug response
}

\author{
Ian R. Phillips ${ }^{1}$ and Elizabeth A. Shephard ${ }^{2}$ \\ ${ }^{1}$ School of Biological and Chemical Sciences, Queen Mary, University of London, Mile End Road, London E1 4NS, UK \\ ${ }^{2}$ Department of Biochemistry and Molecular Biology, University College London, Gower Street, London WC1E 6BT, UK \\ Corresponding author: Shephard, E.A. (e.shephard@ucl.ac.uk).
}

\begin{abstract}
Flavin-containing monooxygenases (FMOs) metabolize numerous foreign chemicals, including drugs, pesticides and dietary components and, thus, mediate interactions between humans and their chemical environment. We describe the mechanism of action of FMOs and insights gained from the structure of yeast FMO. We then concentrate on the three FMOs (FMOs 1, 2 and 3) that are most important for metabolism of foreign chemicals in humans, focusing on the role of the FMOs and their genetic variants in disease and drug response. Loss-of-function mutations of $\mathrm{FMO} 3$ cause the disorder trimethylaminuria. More common variants that decrease enzyme activity are associated with increased drug efficacy. Most humans are homozygous for a nonsense mutation that inactivates FMO2. But a substantial proportion of sub-Saharan Africans express functional FMO2 and, thus, are predicted to respond differently to drugs and other foreign chemicals.
\end{abstract}

\section{Introduction}

Humans daily ingest a vast array of potentially harmful foreign chemicals. Fortunately, we possess enzymes that enable us to metabolize these chemicals into forms that are generally less toxic and more readily excreted. One such family of enzymes, the flavin-containing monooxygenases (FMOs, EC 1.14.13.8), catalyzes the oxygenation of a wide range of foreign chemicals, including therapeutic drugs, pesticides and dietary-derived compounds [1-3]. Thus, FMOs play an important protective role by mediating interactions between humans and their chemical environment.

FMOs have been long overlooked, in comparison with the cytochromes P450 (CYPs), a larger and more extensively studied family of detoxification enzymes. However, characterization of members of the FMO family in humans and of the metabolic consequences of rare mutations and common polymorphic variants of FMOs has led to a growing appreciation of the pharmacological and toxicological significance of these enzymes [3,4]. In contrast to CYPs FMOs are generally not induced or inhibited by foreign chemicals, suggesting that therapeutic agents that are metabolized predominantly by FMOs would be less prone to drug-drug interactions [2]. Observed interindividual differences in the amount or activity of FMOs are, therefore, more likely due to genetic than environmental factors [1,3,4]. An understanding of the pharmacogenetics of FMOs would help predict interindividual variation in drug response and efficacy and, thus, contribute to drug development. This review considers the role of FMOs and their genetic variants in disease and drug response.

\section{Mechanism and structure}

For catalysis FMOs require flavin adenine dinucleotide (FAD) as a prosthetic group, NADPH as a cofactor and molecular oxygen as a cosubstrate $[5,6]$. In contrast to CYPs FMOs accept reducing equivalents directly from NADPH and, thus, do not require accessory proteins. Preferred substrates contain, as the site of oxygenation, a soft nucleophilic heteroatom such as nitrogen, sulfur, selenium or phosphorous. Products of FMO-catalyzed reactions are generally more polar and excretable and less toxic or pharmacologically active than the parent compound. FMOs have an unusual mechanism of action (Figure 1), which enables them to activate oxygen, in the form of stable hydroperoxyflavin, in the absence of bound substrate [5,6]. The enzyme, thus, is present in an activated form that can oxidize any soft nucleophile capable of gaining access to the active site. This unusual feature of FMOs accounts for their broad substrate range.

The structure of a FMO of the yeast Schizosaccharomyces pombe recently has been determined [7] (Figure 2a). The protein is composed of two structural domains, a large domain and a small, or insertion, domain, with a channel between them. FAD only interacts with the large domain, the flavin being exposed to the channel. NADPH binds, via its adenine group, to the insertion domain, its nicotinamide group stacking, via hydrogen-bonding, to the exposed isoalloxazine ring of FAD. The crystal structure of the yeast enzyme-substrate complex indicates that the enzyme does not form the expected quaternary complex with FAD, NADPH and substrate. Instead, the substrate (methimazole) apparently competes with, and replaces, NADPH; it stacks with the isoalloxazine ring, making no direct contact with the polypeptide (Figure $2 \mathrm{~b}$ ).

The structural evidence indicates striking differences in catalytic mechanism from that deduced from spectrophotometric and kinetic studies of mammalian FMOs $[5,6,8]$. The yeast enzyme is predicted to exist in the cell as an enzyme- $\mathrm{FADH}_{2}-\mathrm{NADP}^{+}$complex, and there is no evidence for a stable hydroperoxyflavin form in the absence of substrate. Instead, the structure indicates simultaneous binding of substrate and molecular oxygen, with the latter being positioned near the isoalloxazine ring by H-bonding to Asn91, the only amino-acid residue for 
which there is evidence of direct involvement in catalysis (Figure 2b). This suggests that in the yeast FMO, dioxygen acceptance occurs only in the presence of substrate and that 4a-hydroperoxyflavin represents a transient intermediate, rather than the stable intermediate, in mammalian FMOs (see Figure 1).

It is not clear to what extent the mechanism deduced from the structure of the cytosolic yeast enzyme reflects that of mammalian FMOs, which are located in the membranes of the endoplasmic reticulum. The substrate range of the $S$. pombe FMO is not known. However, the substrate range of the FMO of another yeast, Saccharomyces cerevisiae, is more restricted than that of mammalian FMOs [9], reflecting its role in maintaining redox balance in the cell [10]. The ability of mammalian FMOs to metabolize a broad range of foreign chemicals might be a consequence of differences in catalytic mechanism that arose during evolution.

\section{The FMO family of humans}

Initial research on the FMOs of human was driven largely by the quest to identify the molecular basis of the inherited disorder trimethylaminuria (TMAuria) (see below). We now know that humans possess five functional FMO genes (FMO1 to FMO5) [11-13]. FMO1 to FMO4 are clustered on chromosome 1 at q24.3 [13], along with a pseudogene, FMO6P [13,14]. FMO5 is located $\sim 26 \mathrm{Mbp}$ closer to the centromere, at 1q21.1 [13]. A second FMO gene cluster, composed of five pseudogenes (FMO7P to $F M O 11 P$ ), is present at $1 \mathrm{q} 24.2$ [13]. The five functional FMO genes are thought to have arisen, via duplications of an ancestral gene, some 210-275 million years ago [13]. The proteins they encode have $51 \%$ to $57 \%$ amino acid sequence identity [12]. Of these, FMOs 1 and 3 have been implicated in disease and FMOs 1, 2 and 3 are the most important in the metabolism of foreign chemicals [1-3]. This review concentrates on the roles of FMOs 1, 2 and 3 and their genetic variants in disease and drug metabolism.

\section{FMOs and disease}

\section{FMO3 and TMAuria}

Loss-of-function mutations in FMO3 cause the disorder TMAuria [15,16], which is inherited in a recessive manner. Affected individuals are unable to metabolize the smelly free amine trimethylamine (TMA) to its nonodorous $N$-oxide $[17,18]$. TMA is derived from dietary precursors, such as choline and lecithin, by the action of gut bacteria. The disorder is sometimes called fish-odor syndrome because sufferers secrete excessive amounts of TMA in their breath, sweat and urine, which imparts an odor resembling that of rotting fish. Although no physical symptoms are associated with TMAuria, the unpleasant odor characteristic of the disorder often results in psychological and social problems, in extreme cases leading to clinical depression and suicidal tendencies [17].

The incidence of heterozygotes in British Caucasians is about $1 \%$, but in other populations it might be considerably higher [19]. More than 30 distinct causative mutations have been identified (Figure 3) $[3,18,20]$. Some prevent binding of FAD and, thus, impair assembly of the holoenzyme, whereas others affect kinetic competency
[21]. TMAuria sufferers lack functional FMO3. Consequently, their ability to metabolize FMO3 drug substrates, such as the nonsteroidal anti-inflammatory drug benzydamine, will be impaired [22].

Historical references to individuals who might have suffered from TMAuria include the description of Satyavati, a young woman who smelled of rotting fish, in the 'Mahabharata', an Indian epic compiled in about AD 400, and Trinculo's description of Caliban in Shakespeare's 'The Tempest' [17]. Despite understanding its genetic basis, the disorder has no cure and causes much misery to those who are affected. Treatment strategies rely on dietary restriction of TMA precursors and suppression of intestinal production of TMA $[17,18,23-25]$.

\section{Associations of FMO1 with disease}

Gene expression profiling indicates that the FMO1 gene is down-regulated in the spinal cord of patients with the neurodegenerative disease amyotrophic lateral sclerosis (ALS) [26]. A recent study [27] found that two singlenucleotide polymorphisms (SNPs), located within the $3^{\prime}$ UTR of the mRNA, were overrepresented in female, but not male, sporadic ALS patients. In contrast FMO1 expression is increased in the myocardial tissue of patients with atrial fibrillation (AF) [28]. Although these findings are intriguing, the potential role of FMO1 in the etiology of these diseases remains to be established.

\section{FMOs and drug metabolism}

Tissue distribution and regulation of expression

Although FMO1 and FMO3 have some substrates in common, for example tamoxifen [29] and thiacetazone [30], their tissue distribution differs. Consequently, the relative contribution of a particular FMO to the metabolism of a drug will be tissue dependent.

In adult humans the main site of expression of FMO1 is the kidney [12,31-33]. In this organ the amount of FMO1 [33] exceeds that of the total content of CYPs [34] and approaches that observed in the liver for the major hepatic CYP CYP3A4 [35]. FMO1 is, thus, likely to play an important role in the renal metabolism of drugs. The FMO1 gene is expressed in fetal human liver [12,3133,36], but its expression is extinguished just after birth [36]. Thus, in the adult human FMO1 makes no contribution to the hepatic metabolism of drugs. The lack of FMO1 expression in adult human liver [12,31-33,36] is in contrast to all other mammals investigated, in which FMO1 represents a major hepatic form of the enzyme. LINE-1 elements upstream of the core liver promoter of FMO1 act as powerful transcription repressors, which might account for the silencing of the gene in the adult human liver [37]. Expression of FMO1 in adult human extrahepatic tissues can be explained by the use of alternative, tissue-specific promoters [13,37].

Of the FMOs involved in drug metabolism, FMO3 is the main form present in the adult human liver [12,13,32], which is not the case in other mammals investigated. The age at which the gene is switched on varies from birth to up to two years old, but by 10 months of age most individuals are expressing the enzyme [36]. FMO3 
expression continues to increase until adulthood [36], by which stage the enzyme is present in amounts similar to those of CYP2C9, which represents about $20 \%$ of total hepatic CYPs [35].

The delay between silencing of the FMO1 gene and the full activation of FMO3 means that during the first year of life many children have no, or very little, drugmetabolizing FMOs in their liver.

\section{Genetic Polymorphisms and Drug Metabolism}

\section{FMO3}

FMO3 metabolizes a number of drugs, including the antipsychotics amphetamine and clozapine, the monoamine oxidase $\mathrm{B}$ inhibitor deprenyl, the psychostimulant metamphetamine, the antiestrogen tamoxifen, the antituberculars ethionamide and thiacetazone and the activated form of the nonsteroidal anti-inflammatory agent sulindac sulfide (reviewed in Refs [1,3]).

FMO3 is highly polymorphic, with as many as 15 nonsynonymous SNPs identified, many of which are present at relatively high frequency (reviewed in Refs $[3,4]$ ). Individually, most of the SNPs have little or no effect on enzyme activity [1-4,38]. Three, however, do affect activity: g. $11177 \mathrm{C}>\mathrm{A}(\mathrm{N} 61 \mathrm{~K})$ causes a dramatic decrease in enzyme activity towards four different substrates [39], g.21599T $>$ C(L360P) increases catalytic activity 2- to 5-fold [40] and is the only SNP known to increase FMO3 activity and g.15550C $>$ T(R205C) has a moderate effect on enzyme activity but, interestingly, exhibits substrate inhibition of sulindac sulfide $S$ oxygenation [41]. However, each of the three SNPs is present in low frequency and/or is restricted to single population groups

(http://www.ncbi.nlm.nih.gov/projects/SNP)].

Consequently, they have limited significance for the general population.

In Europeans and Asians the two most common SNPs, g.15167G $>$ A(E158K) [15,42] and g.21443A $>$ G(E308G) [16], are often linked, occurring on the same chromosome [4346]. The compound variant (E158K/E308G) can result in a reduction of enzyme activity, the extent of which depends on the substrate [2-4,38,40,41,46]. Homozygotes for $158 \mathrm{~K} / 308 \mathrm{G}$ can exhibit symptoms of mild or transient TMAuria [47,48]. Many of the reported cases, however, are of young children, in whom low expression of FMO3 [36] (see above) might contribute to symptoms.

A reduced FMO3 activity can be advantageous. For instance, the FMO3 genotype influenced the clinical outcomes of individuals treated with the nonsteroidal antiinflammatory drug sulindac and who had, or were predisposed to, familial adenomatous polyposis (FAP): those homozygous for $158 \mathrm{~K}$ or $308 \mathrm{G}$ FMO3 variants did not develop polyps [49]. Individuals already suffering from FAP showed polyp regression associated with the FMO3 genotype: only those homozygous or heterozygous for the $158 \mathrm{~K}$ allele or heterozygous for both $158 \mathrm{~K}$ and $308 \mathrm{G}$ alleles showed a reduction in polyp number [50]. Sulindac is a prodrug converted by gut bacteria to its active metabolite, sulindac sulfide, which is then absorbed
[51,52]. FMO3 then catalyzes the oxygenation of sulindac sulfide [53] to its inactive sulfoxide [54]. It is thought that enhanced sulindac efficacy in patients with E158K and E308G variants is the result of decreased oxidation of sulindac sulfide by FMO3, leading to an increase in the amount of active drug.

Genetic variants that affect the amount of FMO3 produced also have been identified [55]. Seven SNPs were identified in the $5^{\prime}$-flanking sequence of $F M O 3$, from which 15 haplotypes were inferred. In comparison with the reference sequence, one of the variant haplotypes increased transcription of a FMO3-reporter gene construct 8 -fold. If the increased activity observed in vitro is reflected in vivo, individuals with this haplotype, particularly homozygotes, would be expected to metabolize FMO3 substrates more rapidly, leading to a potential reduction in drug efficacy. Although this haplotype is present in relatively high frequency, it is often associated with the linked variant E158K/E308G [39]. In this case an increase in the amount of protein produced, via promoter SNPs, might compensate for an enzyme with lower activity due to coding SNPs. The presence of such linked SNPs might explain some of the discrepancies between results of analyses of mutant enzyme activity in vitro and drug metabolism in vivo. Two of the promoter-region haplotypes resulted in very low transcriptional activity in vitro [55]. However, each of these is present at low frequency in a single population group and, thus, would have little relevance for the general population.

Analysis of genetic variation in FMO3 provides strong evidence that the gene has been the subject of balancing natural selection [56]. Balancing selection results in two or more haplotypes being maintained at higher frequencies than expected under a neutral model of evolution. In addition to the ancestral FMO3 haplotype, two variant haplotypes have been maintained. One contains both E158K and E308G SNPs and, thus, would encode an enzyme with a reduced activity. The other contains the $5^{\prime}$ flanking SNPs that cause an 8-fold increase in promoter activity (see above). The natural selection of haplotypes that increase or decrease the amount or activity of FMO3 suggests that the resulting metabolic versatility conferred an advantage during human evolution.

\section{FMO2}

FMO2 is an example of a gene that is en route to becoming a pseudogene in humans. Most individuals are homozygous for a $\mathrm{C}>\mathrm{T}$ mutation that changes a glutamine codon at position 472 to a stop codon [57]. The resultant allele $(F M O 2 * 2 A)$ encodes a truncated polypeptide that lacks 64 residues from its carboxy-terminus and is catalytically inactive [57]. The nonsense mutation g.23238C $>\mathrm{T}(\mathrm{Q} 472 \mathrm{X})$ is not present in our closest relatives, chimpanzee (Pan troglodytes) and gorilla (Gorilla gorilla) [57], and, therefore, arose in the human lineage after the divergence of humans and chimps. The FMO2*2A allele subsequently spread to attain a frequency of essentially $100 \%$ in all populations, with the exception of those of recent African descent in which the ancestral allele $\left(F M O 2^{*} 1\right)$, which encodes a full-length polypeptide, is present at appreciable frequencies $[57,58]$. The presence of 
full-length active FMO2 in lung microsomes from an $F M O 2^{*} 1$ individual has been confirmed [59]. The FMO2*1 allele is present throughout sub-Saharan Africa, in some regions attaining frequencies as high as $26 \%$, with almost $50 \%$ of individuals having at least one $F M O 2^{*} 1$ allele [60].

Several other nonsynonomous SNPs have been identified [3,4,61], some of which abolish catalytic activity [62]. Haplotype analysis indicates that the loss-of-function SNPs occur on the $F M O 2 * 2 A$ allele and, thus, are associated with a protein that is already inactive [62]. Individuals who possess a $F M O 2^{*} 1$ allele, thus, are likely to express a catalytically active protein. The distribution of $F M O 2^{*} 1$ and $F M O 2^{*} 2 A$ alleles among world populations has implications for interethnic and, in African populations, interindividual variation in response to drugs and in susceptibility to toxic chemicals that are substrates of FMO2, particularly those for which the lung is the target organ or route of entry.

FMO2 catalyzes the oxygenation of a number of sulfurcontaining environmental chemicals. It detoxifies the thioether-containing organophosphate insecticides phorate and disulfoton [63] but converts thiourea and some of its derivatives to sulfenic or sulfinic acid metabolites, which are more toxic than the parent compound [64]. Thus, individuals who express functional FMO2 might have a reduced risk of toxicity if exposed to organophosphates but a greater risk from exposure to thioureas. Although little is known about drug substrates for human FMO2, it recently has been found to be capable of oxygenating the antitubercular drugs ethionamide and thiacetazone (A. Francois et al., unpublished), which has consequences for the efficacy and toxicity of these drugs in African populations.

\section{FMO1}

Of the three FMOs described in this review, FMO1 has the broadest substrate range, which includes the antidepressant imipramine, the antipsychotic chlorpromazine, the gastroprokinetic itopride and the antihistamine olopatadine (for a more complete list of FMO1 substrates, see Refs [1,3]).

In contrast to FMO2 and FMO3, few nonsynonymous SNPs have been identified in FMO1 [3,4,61] and each is confined to a single population group in which it is present at low frequency [3]. Three of the four SNPs tested had little or no effect on catalytic activity [65]. The other, g.27362C > T(R502X), which was identified in only 1 of 100 chromosomes [61], completely abolished activity towards methimazole but had no effect on activity towards three other substrates [65].

Interindividual variations in the amount of FMO1 protein have been reported $[4,36]$. A number of SNPs have been identified close to the fetal liver-specific FMO1 promoter [66]. Only one, g.-9536C $>$ A, was located in an element previously implicated in transcriptional regulation of the gene [67]. This SNP, located in a YY1 element, prevented binding of the transcription factor and decreased promoter activity in vitro [66]. However, the SNP (the $F M O 1^{*} 6$ allele), which is present in relatively high frequency, was not correlated with lower FMO1 expression in fetal liver in vivo [66]. No SNPs yet have been identified in the alternative $F M O 1$ promoters active in extrahepatic tissues [37].

An indication of the importance of FMO1 in drug detoxification is demonstrated by a recent study on Fmo1deficient mice (D. Hernandez et al., unpublished). When treated with the antidepressant imipramine, which is normally converted to its $N$-oxide by FMO1, the mice display an adverse reaction. This is accompanied by a marked increase in the amount of parent drug in the serum and kidneys and higher amounts of the metabolite desipramine in the brain, indicating that in the absence of FMO1 more of the drug is metabolized via CYP-mediated pathways.

\section{Concluding remarks}

The main features of the three FMOs involved in the metabolism of foreign chemicals are summarized in Table 1. FMOs catalyze the oxygenation of a wide variety of drugs and other foreign chemicals. The broad substrate range of the enzymes is a consequence of their unusual mechanism of action. Several genetic variants of FMOs of human have been identified. Loss-of-function mutations in FMO3 cause TMAuria, a disorder characterized by an unpleasant body odor that often results in psychological and social problems. More common polymorphic variants of FMO3, particularly when present in cis, can affect the amount or activity of the enzyme and, hence, alter drug efficacy. Some of these variants have been maintained during human evolution and, thus, might confer a selective advantage.

Most people have a nonsense mutation that renders FMO2 inactive. However, a substantial minority of subSaharan Africans express functional FMO2 and, thus, might be at greater risk of toxicity when exposed to chemicals, such as thioureas, that the enzyme converts into toxic products. Although FMO1 has the widest range of drug substrates, its expression in the human liver is silenced at birth and its contribution to drug metabolism in the adult is, therefore, restricted to extrahepatic tissues.

Recent work on FMOs of humans, particularly the identification of genetic variants and the consequences of these for disease and drug response, has increased awareness of the pharmacological and toxicological significance of these enzymes. Future work should help define the contributions of FMOs to multipathway drug metabolism and enable us to predict how genetic variation in FMOs might lead to adverse drug reactions or enhanced drug efficacy.

\section{References}

1 Krueger, S.K. and Williams, D.E. (2005) Mammalian flavincontaining monooxygenases: structure/function, genetic polymorphisms and role in drug metabolism. Pharmacol. Ther. 106, 357-387

2 Cashman, J.R. and Zhang, J. (2006) Human flavin-containing monooxygenases. Annu .Rev. Pharmacol. Toxicol. 46, 65-100

3 Phillips, I.R. et al. (2007) The flavin-containing monooxygenases (FMOs): genetic variation and its consequences for the metabolism of therapeutic drugs. Curr. Pharmacogen. 5, 292-313

4 Koukouritaki, S.B. and Hines, R.N. (2005) Flavin-containing monooxygenase genetic polymorphism: impact on chemical metabolism and drug development. Pharmacogenomics 6, 807-822 
5 Ziegler, D.M. (1993) Recent studies on the structure and function of multisubstrate flavin-containing monooxygenases. Annu. Rev. Pharmacol. Toxicol. 33, 179-199

6 Ziegler, D.M. (2002) An overview of the mechanism, substrate specificities, and structure of FMOs. Drug Metab. Rev. 34, 503-511

7 Eswaramoorthy, S. et al. (2006) Mechanism of action of a flavincontaining monooxygenase. Proc. Natl. Acad. Sci. U. S. A. 103, 9832-9837

8 Poulsen, L.L. and Ziegler, D.M. (1979) The liver microsomal FADcontaining monooxygenase. Spectral characterization and kinetic studies. J. Biol. Chem. 254, 6449-6455

9 Suh, J.K. et al. (1996) Molecular cloning and kinetic characterization of a flavin-containing monooxygenase from Saccharomyces cerevisiae. Arch. Biochem. Biophys. 336, 268-274

10 Suh, J.K. et al. (2000) Redox regulation of yeast flavin-containing monooxygenase. Arch. Biochem. Biophys. 381, 317-322

11 Lawton, M.P. et al. (1994) A nomenclature for the mammalian flavin-containing monooxygenase gene family based on amino acid sequence identities. Arch. Biochem. Biophys. 308, 254-257

12 Phillips, I.R. et al. (1995) The molecular biology of the flavincontaining monooxygenases of man. Chem. Biol. Interact. 96, 17-

13 Hernandez, D. et al. (2004) Organization and evolution of the flavin-containing monooxygenase genes of human and mouse: identification of novel gene and pseudogene clusters. Pharmacogenetics 14, 117-130

14 Hines, R.N. et al. (2002) Alternative processing of the human FMO6 gene renders transcripts incapable of encoding a functional flavin-containing monooxygenase. Mol. Pharmacol. 62, 320-325

15 Dolphin, C.T. et al. (1997) Missense mutation in flavin-containing mono-oxygenase 3 gene, FMO3, underlies fish-odour syndrome. Nat. Genet. 17, 491-494

16 Treacy, E.P. et al. (1998) Mutations of the flavin-containing monooxygenase gene (FMO3) cause trimethylaminuria, a defect in detoxication. Hum. Mol. Genet. 7, 839-845

17 Mitchell, S.C. and Smith, R.L. (2001) Trimethylaminuria: the fish malodor syndrome. Drug Metab. Dispos. 29, 517-521

18 Phillips, I.R. and Shephard, E.A. (2007) Trimethylaminuria. In: GeneReviews at GeneTests: Medical Genetics Information Resource (database online). Copyright, University of Washington, Seattle. 1997-2007. Available at http://www.genetests.org.

19 Mitchell, S.C. et al. (1997) Studies on the discontinuous Noxidation of trimethylamine among Jordanian, Ecuadorian and New Guinean populations. Pharmacogenetics 7, 45-50

20 Hernandez, D. et al. (2003) Trimethylaminuria and a human FMO3 mutation database. Hum. Mut. 22, 201-213

21 Yeung, C.K. et al. (2007) Functional characterization of genetic variants of human FMO3 associated with trimethylaminuria. Arch. Biochem. Biophys. 464, 251-259

22 Mayatepek, E. et al. (2004) Benzydamine metabolism in vivo is impaired in patients with deficiency of flavin-containing monooxygenase 3. Pharmacogenetics 14, 775-777

23 Cashman, J.R. et al. (2003) Biochemical and clinical aspects of the human flavin-containing monooxygenase form 3 (FMO3) related to trimethylaminuria. Curr. Drug. Metab. 4, 151-170

24 Chalmers, R.A. et al. (2006) Diagnosis and management of trimethylaminuria (FMO3 deficiency) in children. J. Inherit. Metab. Dis. 29, 162-172

25 Yamazaki, H. et al. (2004) Effects of the dietary supplements, activated charcoal and copper chlorophyllin, on urinary excretion of trimethylamine in Japanese trimethylaminuria patients. Life Sci. 74, 2739-2747

26 Malaspina, A. et al. (2001) Differential expression of 14 genes in amyotrophic lateral sclerosis spinal cord detected using gridded cDNA arrays. J. Neurochem. 77, 132-145

27 Cereda, C. et al. (2006) Increased incidence of FMO1 gene single nucleotide polymorphisms in sporadic amyotrophic lateral sclerosis. Amyotroph. Lateral Scler. 7, 227-234

28 Kim, Y.H. et al. (2003) Gene expression profiling of oxidative stress on atrial fibrillation in humans. Exp. Mol. Med. 35, 336-349

29 Krueger, S.K. et al. (2006) The role of flavin-containing monooxygenase (FMO) in the metabolism of tamoxifen and other tertiary amines. Drug Metab. Rev. 38, 139-147
30 Qian, L. and Ortiz de Montellano, P.R. (2006) Oxidative activation of thiacetazone by the Mycobacterium tuberculosis flavin monooxygenase EtaA and human FMO1 and FMO3. Chem. Res. Toxicol. 19, 443-449

31 Dolphin, C. et al. (1991) Cloning, primary sequence, and chromosomal mapping of a human flavin-containing monooxygenase (FMO1). J. Biol. Chem. 266, 12379-12385

32 Dolphin, C.T. et al. (1996) Differential developmental and tissuespecific regulation of expression of the genes encoding three members of the flavin-containing monooxygenase family of man, FMO1, FMO3 and FM04. Eur. J. Biochem. 235, 683-689

33 Yeung, C.K. et al. (2000) Immunoquantitation of FMO1 in human liver, kidney, and intestine. Drug Metab. Dispos. 28, 1107-1111

34 Jakobsson, S.V. and Cintig, D.L. (1973) Studies on the cytochrome $\mathrm{P}$-450-containing mono-oxygenase system in human kidney cortex microsomes. J. Pharmacol. Exp. Ther. 185, 226-234

35 Shimada, T. et al. (1994) Interindividual variations in human liver cytochrome P-450 enzymes involved in the oxidation of drugs, carcinogens and toxic chemicals: studies with liver microsomes of 30 Japanese and 30 Caucasians. J. Pharmacol. Exp. Ther. 270, $414-423$

36 Koukouritaki, S.B. et al. (2002) Human hepatic flavin-containing monooxygenases 1 (FMO1) and 3 (FMO3) developmental expression. Pediatr. Res. 51, 236-243

37 Shephard, E.A. et al. (2007) Alternative promoters and repetitive DNA elements define the species-dependent tissue-specific expression of the FMO1 genes of human and mouse. Biochem. J. 406, 491-499

38 Cashman, J.R. (2004) The implications of polymorphisms in mammalian flavin-containing monooxygenases in drug discovery and development. Drug Discov. Today 9, 574-581

39 Koukouritaki, S.B. et al. (2007) Identification and functional analysis of common human flavin-containing monooxygenase 3 genetic variants. J. Pharmacol. Exp. Ther. 320, 266-273

40 Lattard, V. et al. (2003) Two new polymorphisms of the FMO3 gene in Caucasian and African-American populations: comparative genetic and functional studies. Drug Metab. Dispos. 31, 854-860

41 Shimizu, M. et al. (2007) Effect of genetic variants of the human flavin-containing monooxygenase 3 on $\mathrm{N}$ - and S-oxygenation activities. Drug Metab. Dispos. 35, 328-330

42 Brunelle, A. et al. (1997) Characterization of two human flavincontaining monooxygenase (form 3) enzymes expressed in Escherichia coli as maltose binding protein fusions. Drug Metab. Dispos. 25, 1001-1007

43 Sachse, C. et al. (1999) Flavin monooxygenase 3 (FMO3) polymorphism in a white population: allele frequencies, mutation linkage, and functional effects on clozapine and caffeine metabolism. Clin. Pharmacol. Ther. 66, 431-438

44 Kang, J.H. et al. (2000) Phenotypes of flavin-containing monooxygenase activity determined by ranitidine $\mathrm{N}$-oxidation are positively correlated with genotypes of linked FM03 gene mutations in a Korean population. Pharmacogenetics $10,67-78$

45 Cashman, J.R. et al. (2001) Population distribution of human flavin-containing monooxygenase form 3: gene polymorphisms. Drug Metab. Dispos. 29, 1629-1637

46 Park, C.S. et al. (2002) Ethnic differences in allelic frequency of two flavin-containing monooxygenase 3 (FMO3) polymorphisms: linkage and effects on in vivo and in vitro FMO activities. Pharmacogenetics 12, 77-80

47 Mayatepek, E. and Kohlmüller, D. (1998) Transient trimethylaminuria in childhood. Acta. Paediatr. 87, 1205-1207

48 Zschocke, J. et al. (1999) Mild trimethylaminuria caused by common variants in FMO3 gene. Lancet 354, 834-835

49 Hisamuddin, I.M. et al. (2004) Genetic polymorphisms of human flavin monooxygenase 3 in sulindac-mediated primary chemoprevention of familial adenomatous polyposis. Clin. Cancer Res. 10, 8357-8362

50 Hisamuddin, I.M. et al. (2005) Genetic polymorphisms of flavin monooxygenase 3 in sulindac-induced regression of colorectal adenomas in familial adenomatous polyposis. Cancer Epidemiol. Biomarkers Prev. 14, 2366-2369

51 Duggan, D.E. et al. (1977) Identification of the biologically active form of sulindac. J. Pharmacol. Exp. Ther. 201, 8-13 
52 Etienne, F. et al. (2003) Reduction of Sulindac to its active metabolite, sulindac sulfide: assay and role of the methionine sulfoxide reductase system. Biochem. Biophys. Res. Commun. 312, 1005-1010

53 Hamman, M.A. et al. (2000) Stereoselective sulfoxidation of sulindac sulfide by flavin-containing monooxygenases. Comparison of human liver and kidney microsomes and mammalian enzymes. Biochem. Pharmacol. 60, 7-17

54 Duggan, D.E. et al. (1977) The disposition of sulindac. Clin. Pharmacol. Ther. 21, 326-335

55 Koukouritaki, S.B. et al. (2005) Discovery of novel flavincontaining monooxygenase 3 (FMO3) single nucleotide polymorphisms and functional analysis of upstream haplotype variants. Mol. Pharmacol. 68, 383-392

56 Allerston, C.K. et al. (2007) Molecular evolution and balancing selection in the flavin-containing monooxygenase 3 gene (FMO3). Pharmacogenet. Genomics 17, 827-839

57 Dolphin, C.T. et al. (1998) The flavin-containing monooxygenase 2 gene (FMO2) of humans, but not of other primates, encodes a truncated, nonfunctional protein. J. Biol. Chem. 273, 30599-30607

58 Whetstine, J.R. et al. (2000) Ethnic differences in human flavincontaining monooxygenase 2 (FMO2) polymorphisms: detection of expressed protein in african-americans. Toxicol. Appl. Pharmacol. 168, 216-224

59 Krueger, S.K. et al. (2002) Identification of active flavin-containing monooxygenase isoform 2 in human lung and characterization of expressed protein. Drug Metab. Dispos. 30, 34-41

60 Veeramah, K. et al. The potentially deleterious functional variant $F_{M O 2}{ }^{*} 1$ is at high frequency throughout sub-Saharan Africa. Pharmacogenet Genomics (in press)

61 Furnes, B. et al. (2003) Identification of novel variants of the flavin-containing monooxygenase gene family in African Americans. Drug Metab. Dispos. 31, 187-193

62 Krueger, S.K. et al. (2005) Haplotype and functional analysis of four flavin-containing monooxygenase isoform 2 (FMO2) polymorphisms in Hispanics. Pharmacogenet. Genomics 15, 245256

63 Henderson, M.C. et al. (2004) S-oxygenation of the thioether organophosphate insecticides phorate and disulfoton by human lung flavin-containing monooxygenase 2. Biochem. Pharmacol. 68, 959-967

64 Henderson, M.C. et al. (2004) Human flavin-containing monooxygenase form 2 S-oxygenation: sulfenic acid formation from thioureas and oxidation of glutathione. Chem. Res. Toxicol. 17, $633-640$

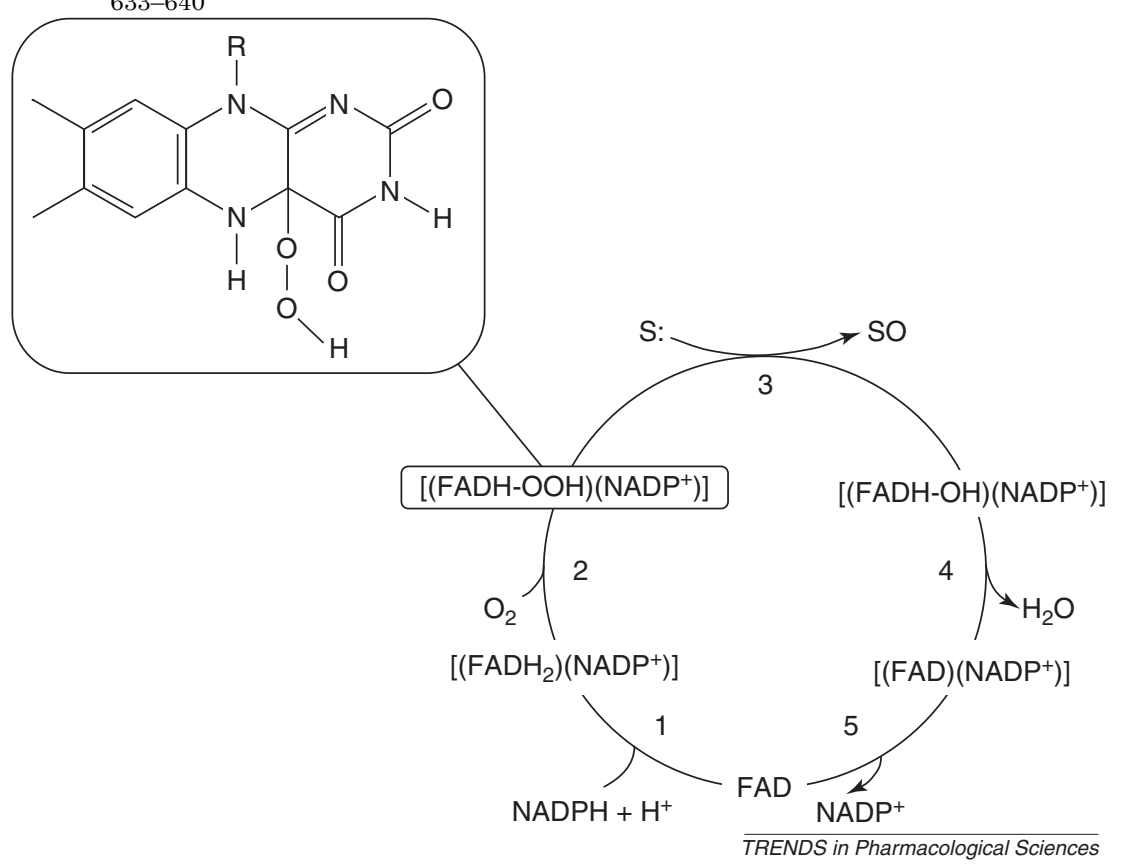

Figure 1. The catalytic cycle of mammalian FMOs. Evidence for the cycle is based on spectrophotometric and kinetic studies (reviewed in Refs [5,6]). The cofactor NADPH binds and reduces the prosthetic group FAD to $\mathrm{FADH}_{2}$, via hydride ion transfer (step 1). Molecular oxygen then binds and is reduced, forming 4a-hydroperoxyflavin (4a-HPF or FADH-
65 Furnes, B. and Schlenk, D. (2004) Evaluation of xenobiotic N- and S-oxidation by variant flavin-containing monooxygenase 1 (FMO1) enzymes. Toxicol. Sci. 78, 196-203

66 Hines, R.N. et al. (2003) Genetic variability at the human FMO1 locus: significance of a basal promoter Yin Yang 1 element polymorphism $\left(\mathrm{FMO}^{*} 6\right)$. J. Pharmacol. Exp. Ther. 306, 12101218

67 Luo, Z. and Hines, R.N. (2001) Regulation of flavin-containing monooxygenase 1 expression by Ying Yang 1 and hepatic nuclear factors 1 and 4. Mol. Pharmacol. 60, 1421-1430

68 Cashman, J.R. et al. (1999) N-oxygenation of amphetamine and methamphetamine by the human flavin-containing monooxygenase (form 3): role in bioactivation and detoxication. $J$.

69 Szoko, E. et al. (2004) Assessment of the N-oxidation of deprenyl, methamphetamine, and amphetamine enantiomers by chiral capillary electrophoresis: an in vitro metabolism study. Electrophoresis 25, 2866-2875

70 Tugnait, M. et al. (1997) N-oxygenation of clozapine by flavincontaining monooxygenase. Drug Metab. Dispos. 25, 524-527

71 Parte, P. and Kupfer, D. (2005) Oxidation of tamoxifen by human flavin-containing monooxygenase (FMO) 1 and FMO3 to tamoxifen-N-oxide and its novel reduction back to tamoxifen by human cytochromes P450 and hemoglobin. Drug Metab. Dispos. 33, 1446-1452

72 Kim, Y.M. and Ziegler, D.M. (2000) Size limits of thiocarbamides accepted as substrates by human flavin- containing monooxygenase 1. Drug Metab. Dispos. 28, 1003-1006

73 Mushiroda, T. et al. (2000) The involvement of flavin-containing monooxygenase but not CYP3A4 in metabolism of itopride hydrochloride, a gastroprokinetic agent: comparison with cisapride and mosapride citrate. Drug Metab. Dispos. 28, 1231-1237

74 Kajita, J. et al. (2002) Effects of olopatadine, a new antiallergic agent, on human liver microsomal cytochrome P450 activities. Drug Metab. Dispos. 30, 1504-1511

75 Dolphin, C.T. et al. (1997) Structural organization of the human flavin-containing monooxygenase 3 gene (FMO3), the favored candidate for fish-odor syndrome, determined directly from genomic DNA. Genomics 46, 260-267

76 Dolphin, C.T. et al. (2000) Compound heterozygosity for missense mutations in the flavin-containing monooxygenase 3 (FM03) gene in patients with fish-odour syndrome. Pharmacogenetics 10, 799807 Pharmacol. Expt. Ther. 288, 1251-1260 
$\mathrm{OOH}$ ) (step 2), which is stabilized by NADP ${ }^{+}$. Steps 1 and 2 are fast. The 4a-HPF, the structure of which is shown, is a stable intermediate and is considered to be the form in which FMO is present in the cell. Oxygenation of substrate $\mathrm{S}$ to $\mathrm{SO}$ occurs via nucleophilic attack on the distal O atom of $4 \mathrm{a}-\mathrm{HPF}$, leaving FAD as the $4 \mathrm{a}-$ hydroxyflavin (FADH$\mathrm{OH}$ ) (step 3). The rate-limiting step is the release of water from the 4a-hydroxyflavin to reform FAD (step 4) and consequently the value of $k_{\text {cat }}$ should be independent of the structure of $\mathrm{S}$. The final step is the release of $\mathrm{NADP}^{+}$(step 5)

(a)

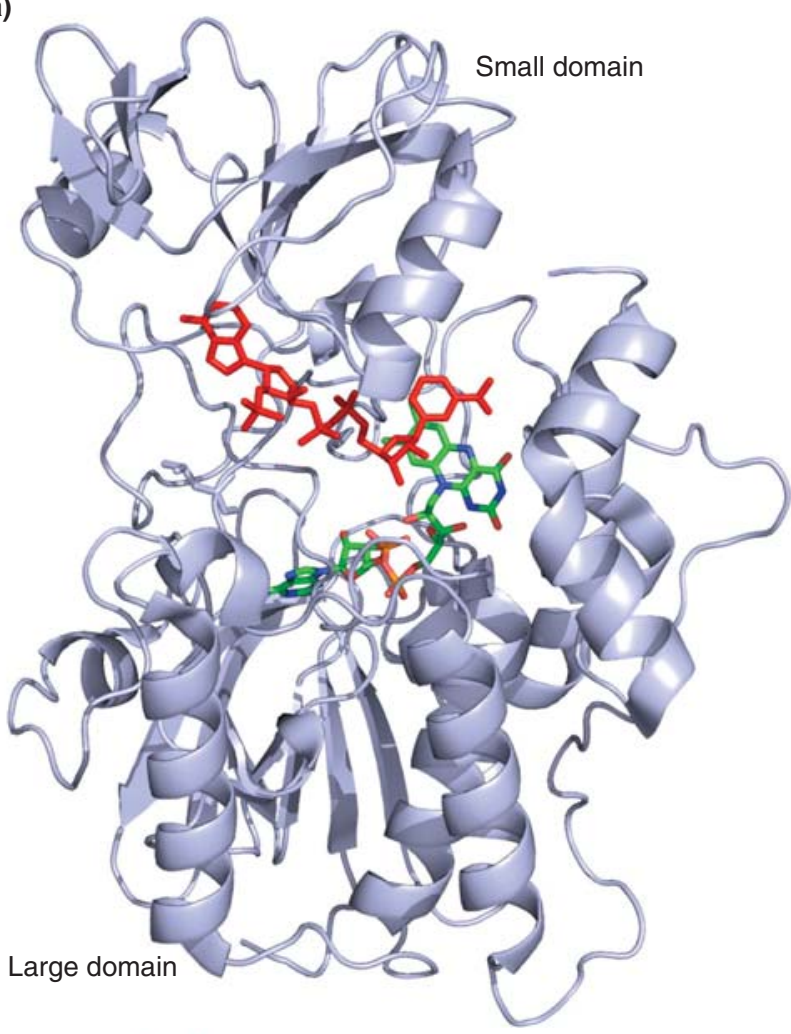

(b)

$\times \checkmark$
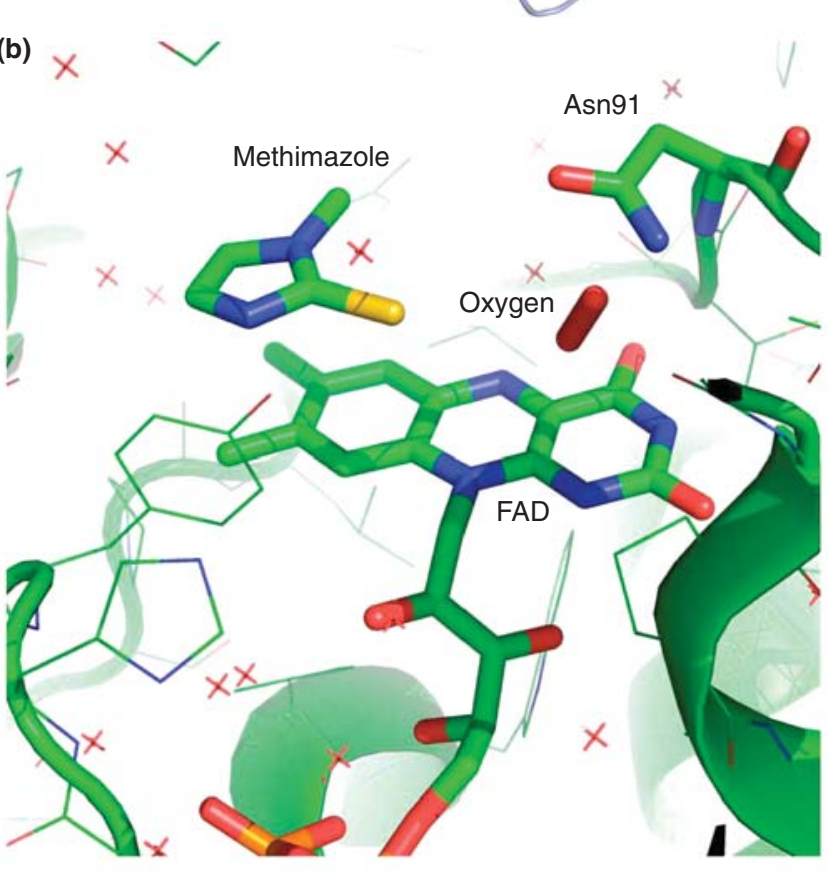

TRENDS in Pharmacological Sciences

Figure 2. Structure of FMO of Schizosaccharomyces pombe. (a) Protein structure (taken from Protein Data Bank ID code 2GV8). The polypeptide is shown as a ribbon representation, and FAD and NADPH as stick models. NADPH is shown in red; for FAD carbon is depicted in green, oxygen in red and nitrogen in blue. (b) Active center depicted from above the isoalloxazine ring of FAD (taken from PDB ID code 2GVC). FAD, Asn at position 91 and the substrate methimazole are shown as stick models. Oxygen is depicted in red, nitrogen in blue and sulfur in yellow. 


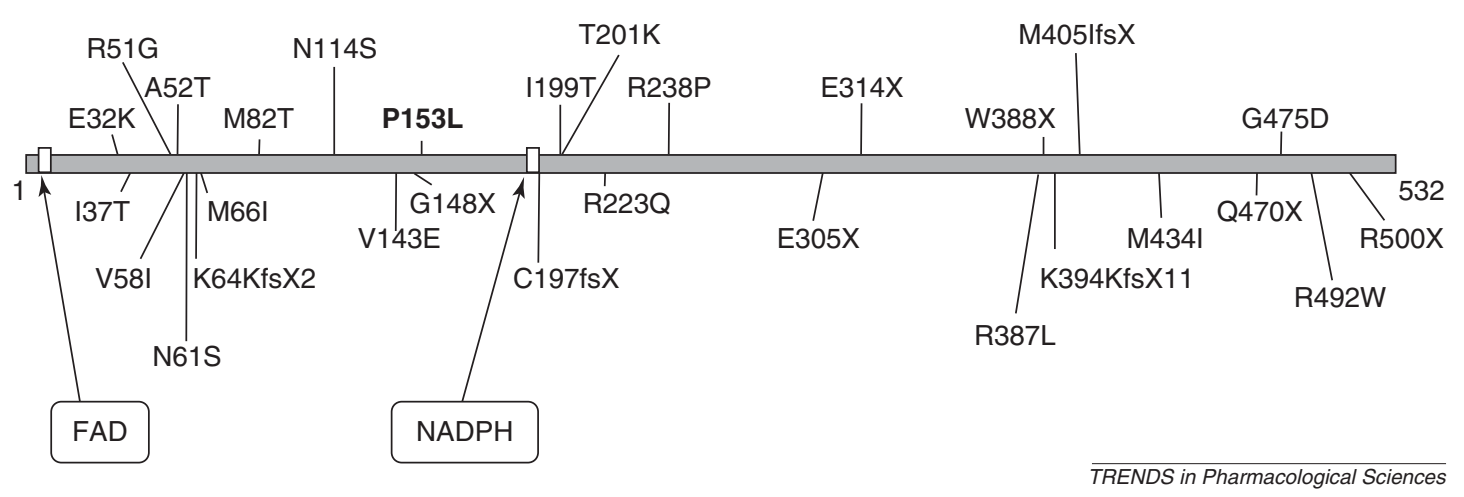

Figure 3. Mutations in FMO3 that cause TMAuria. The grey horizontal bar represents the FMO3 protein. The positions of the FAD- and NADPH-binding sites (white boxes) are indicated by arrows. Mutations in FMO3 are shown above and below the bar. Identification of the mutations was facilitated by determination of the intron-exon structure of the FMO3 gene [75]. P153L (shown in bold) was the first causative mutation identified [15]. This and E305X [16] are the most common. All of the mutations either abolish or severely impair catalytic activity. However, although N61S abolishes TMA N-oxygenation, it does not affect S-oxygenation of methimazole [76]. N61K, a polymorphic variant that severely impairs enzyme activity [39] and, thus, is predicted to cause TMAuria, has not been identified in affected individuals and, therefore, is not included in the figure.

\section{Table 1. Features of the FMOs involved in foreign chemical metabolism}

\begin{tabular}{|c|c|c|c|c|c|}
\hline Gene & $\begin{array}{l}\text { Main site of } \\
\text { expression }\end{array}$ & Links to disease & Examples of substrates $^{1}$ & $\begin{array}{l}\text { Some functionally } \\
\text { significant SNPs }\end{array}$ & SNP effect \\
\hline \multirow[t]{6}{*}{ FMO3 } & \multirow[t]{6}{*}{ Adult liver } & \multirow{2}{*}{$\begin{array}{l}\text { Loss-of-function mutations } \\
\text { cause TMAuria }{ }^{2}\end{array}$} & \multirow{6}{*}{$\begin{array}{l}\text { amphetamine }[68,69] \\
\text { benzydamine [22] } \\
\text { ethionamide [1] } \\
\text { clozapine [70] } \\
\text { deprenyl [69] } \\
\text { metamphetamine }[68,69] \\
\text { tamoxifen [71] } \\
\text { thiacetazone [30] } \\
\text { sulindac sulfide [53] }\end{array}$} & $\mathrm{N} 61 \mathrm{~K}$ & Activity decreased or abolished [39] \\
\hline & & & & R205C & Activity moderately decreased [41] \\
\hline & & \multirow{4}{*}{$\begin{array}{l}\text { Adverse drug reactions or } \\
\text { increased drug efficacy }\end{array}$} & & & \\
\hline & & & & L360P & Activity increased [40] \\
\hline & & & & E158K/E308G & $\begin{array}{l}\text { Activity moderately decreased [2- } \\
4,38,40,41,46] \\
\text { Can cause transient TMAuria in } \\
\text { children }[47,48] \\
\text { Increased efficacy of sulindac } \\
{[49,50]}\end{array}$ \\
\hline & & & & Various promoter SNPs & $\begin{array}{l}\text { Either increase or decrease } \\
\text { expression [55] }\end{array}$ \\
\hline FMO2 & Lung & $\begin{array}{l}\text { Response to drugs and } \\
\text { environmental chemicals }\end{array}$ & $\begin{array}{l}\text { disulfoton [63] } \\
\text { phorate [63] } \\
\text { thiourea-based drugs [64] }\end{array}$ & $\mathrm{Q} 472 \mathrm{X}^{3}$ & Activity abolished [57] \\
\hline \multirow[t]{3}{*}{ FMO1 } & $\begin{array}{l}\text { Fetal liver } \\
\text { Fetal kidney } \\
\text { Adult kidney }\end{array}$ & $\begin{array}{l}\text { Decreased expression in } \\
\text { spinal cord in ALS }\end{array}$ & $\begin{array}{l}\text { chlorpromazine [72] } \\
\text { imipramine [72] } \\
\text { itopride [73] }\end{array}$ & $\mathrm{R} 502 \mathrm{X}$ & $\begin{array}{l}\text { Substrate-dependent abolition of } \\
\text { activity [65] }\end{array}$ \\
\hline & \multirow[t]{2}{*}{ Small intestine } & $\begin{array}{l}\text { Increased expression in } \\
\text { mycocardium in AF }\end{array}$ & $\begin{array}{l}\text { olopatidine [74] } \\
\text { tamoxifen [71] } \\
\text { thiacetazone [30] }\end{array}$ & Promoter SNPs & Decrease expression [66] \\
\hline & & Response to drugs & & & \\
\hline
\end{tabular}

1 In many cases FMO is not the only enzyme involved in the metabolism of the chemical in vivo,

${ }^{2}$ More than 30 causative mutations have been identified $[3,18,20]$,

${ }^{3}$ Other SNPs have been identified, but occur on a Q472X background [62]. 